\title{
Ricci-Flat Metrics on Vector Bundles Over Flag Manifolds
}

\author{
Ismail Achmed-Zade ${ }^{1,2}$, Dmitri Bykov ${ }^{1,2,3}$ \\ ${ }^{1}$ Max-Planck-Institut für Physik, Föhringer Ring 6, 80805 Munich, Germany. \\ E-mail: I.AchmedZade@ physik.uni-muenchen.de; bykov@mpp.mpg.de; bykov@mi-ras.ru; \\ dmitri.v.bykov@gmail.com \\ 2 Arnold Sommerfeld Center for Theoretical Physics, Theresienstrasse 37, 80333 Munich, Germany \\ 3 Steklov Mathematical Institute of Russian Academy of Sciences, Gubkina str. 8, Moscow, Russia 119991
}

Received: 6 May 2019 / Accepted: 16 February 2020

Published online: 19 May 2020 - () The Author(s) 2020

\begin{abstract}
We construct explicit complete Ricci-flat metrics on the total spaces of certain vector bundles over flag manifolds of the group $S U(n)$, for all Kähler classes. These metrics are natural generalizations of the metrics of Candelas-de la Ossa on the conifold, Pando Zayas-Tseytlin on the canonical bundle over $\mathbb{C P}^{1} \times \mathbb{C P}^{1}$, as well as the metrics on canonical bundles over flag manifolds, recently constructed by van Coevering.
\end{abstract}

\section{Contents}

1. Introduction and Main Result . . . . . . . . . . . . . . . . . . 2310

1.1 The flag manifold . . . . . . . . . . . . . . . . . . . . 2310

1.2 Kähler classes . . . . . . . . . . . . . . . . . . . . 2311

1.3 Total spaces of bundles over flag manifolds . . . . . . . . . . . . . . 2312

1.4 Explicit Kähler metrics on flag manifolds . . . . . . . . . . . . . . 2314

1.5 Ricci-flat metrics on vector bundles . . . . . . . . . . . . . . . . 2315

2. Conifold and Canonical Bundle Over $\mathbb{C P}^{1} \times \mathbb{C P}^{1} \ldots \ldots . . . . . . .2316$

3. Flag Manifolds . . . . . . . . . . . . . . . . . . . 2318

3.1 The canonical bundle . . . . . . . . . . . . . . . . . . 2319

3.2 Generalized Calabi Ansatz for all Kähler classes . . . . . . . . . . . . 2320

3.3 Solution of the Ricci-flatness equation . . . . . . . . . . . . . . . 2322

3.3 .1 Behavior at $\infty \ldots \ldots 2322$

3.3.2 The metric on $X_{K} \quad \ldots \ldots \ldots . \ldots . \ldots . \ldots . \ldots . \ldots 2322$

3.3 .3 The metric on $X_{V} \ldots \ldots \ldots \ldots . \ldots \ldots . \ldots . \ldots . \ldots 2323$

A. Complex Structures on Flag Manifolds . . . . . . . . . . . . . . . . . 2324

B. Calculation of the Determinant of the Metric . . . . . . . . . . . . 2325 


\section{Introduction and Main Result}

The problem of constructing explicit Ricci-flat Kähler metrics is rather complicated. In the compact case no such metrics are known, due to the fact that the condition of Ricciflatness implies the absence of non-parallel Killing vectors [1, Section 1.84]. However, in the non-compact case symmetries may be present, and the metrics are sometimes known in explicit form. The early examples include the Eguchi-Hanson 'gravitational instanton' [2] and its generalizations by Gibbons and Hawking [3]. Another example that will be important for us is the metric of Candelas-de la Ossa [4] on the so-called 'resolved conifold' and its immediate generalization constructed in [5]. Various other metrics are known: those of cohomogeneity one of Stenzel [6] and Nitta [7] as well as the higher-cohomegeneity metrics on manifolds that admit Killing-Yano tensors [8,9]. One can also construct hyperkähler metrics on the cotangent bundle of flag manifolds using the hyperkähler quotient construction of [10]. In the simplest case of the Grassmannians this was elaborated upon in [11]. For the purposes of the present paper, however, it will be sufficient to understand in detail the examples of $[2,4,5]$.

An important feature of the metric of [2] is that it may be thought of as the metric on the total space of the canonical bundle $\mathcal{O}(-2)$ over $\mathbb{C P}^{1}$. In fact, it is a simple application of the so-called Calabi ansatz [12], which allows for the construction of a complete Ricci-flat metric on the canonical bundle of a Kähler-Einstein manifold $\mathcal{M}_{K E}$ of positive curvature-in this case this manifold is simply $\mathbb{C P}^{1}$ with its Fubini-Study (round) metric.

1.1. The flag manifold. An interesting generalization may be obtained by replacing the base manifold $\mathbb{C P}^{1}$ by a manifold of flags in $\mathbb{C}^{n}$. We recall that a flag manifold may be specified by a sequence of increasing integers $0<m_{1}<\cdots<m_{s}=n$ which define the flag

$$
0 \subset L_{1} \subset \cdots \subset L_{s}=\mathbb{C}^{n}
$$

where $L_{k}$ are linear subspaces of $\mathbb{C}^{n}$, such that $\operatorname{dim} L_{k}=m_{k}$. In place of $m_{k}$ we will often use the integers $n_{i}$, defined by

$$
m_{k}=\sum_{i=1}^{k} n_{i}
$$

In these terms, the manifold of flags (1.1) is a homogeneous space

$$
\mathscr{F}_{n_{1}, \ldots, n_{s}}=\frac{G L(n, \mathbb{C})}{P_{m_{1}, \ldots, m_{s}}} \simeq \frac{U(n)}{U\left(n_{1}\right) \times \cdots \times U\left(n_{s}\right)} .
$$

Here $P_{m_{1}, \ldots, m_{s}}$ is a parabolic subgroup of $G L(n, \mathbb{C})$, stabilizing the flag (1.1). In what follows we will sometimes use the short notation $\mathscr{F}$ for the manifold (1.3). The complex geometry of flag manifolds was first studied in the classical work [13].

An important observation is that the first representation in (1.3) defines a complex structure on the flag manifold, whereas from the point of view of the unitary quotient in (1.3) the complex structure is an additional piece of data. This additional data is provided by choosing an ordering of the factors in the denominator, which in turn defines the dimensions of the complex vector spaces $L_{k}$ via (1.2), and hence the parabolic subgroup $P_{m_{1}, \ldots, m_{s}}$. In Appendix A we explain the equivalence of this definition to the one of [14], which is based on Lie algebra theory. Throughout the paper we will assume the complex structure on the flag manifold fixed and defined by the ordering used in (1.2). 
1.2. Kähler classes. Flag manifolds admit Kähler-Einstein metrics, as studied in [14, 15]. The authors of [16] use Calabi's ansatz to construct a Ricci-flat metric on the canonical bundle of the flag manifold, equipped with such a Kähler-Einstein metric. An important characteristic of Calabi's ansatz, that we review in Sect. 2, is that it produces a metric with a fixed Kähler class. On the other hand, by the Calabi-Yau theorem [1719], in the case of a compact manifold with $\mathbf{c}_{1}=0$ there should exist a Ricci-flat metric in every Kähler class. The relevant non-compact generalization of the theorem (to asymptotically-conical spaces) was constructed in [20,21]. One has the following statement:

Theorem. [21, Theorem 5.1] Let $X_{0}$ be an affine variety with only normal isolated singularity at $p$. We assume that the complement $X_{0} \backslash p$ is biholomorphic to the cone $C(S)$ of an Einstein-Sasakian manifold $S$. If there is a resolution of singularity $\pi: X \rightarrow X_{0}$ with trivial canonical line bundle $K_{X}$, then there is a Ricci-flat conical Kähler metric for every Kähler class of $X$.

This can readily be applied to the case of canonical bundles over smooth KählerEinstein Fano varieties (see also [21, Example 6.3]). Given a Fano manifold $M$ (of which a flag manifold is an example), one can construct its projective embedding by sections of the ample anti-canonical bundle $K_{M}^{-1}$. The affine cone over the image of this embedding is the affine variety $X_{0}$ and has an isolated singularity at the origin (since $M$ itself is smooth). This variety is the total space of $K_{M}$ with the zero section contracted. It admits a cone metric $C(S)$, with Kähler potential $k_{c}=|u|^{2} e^{r_{0}}$, where $r_{0}$ is the Ricci potential of $M$ and $u$ is the coordinate in the fiber. The metric is Ricci-flat due to the fact that $M$ is Kähler-Einstein (for a review of the relation between Ricci-flat cones, Kähler-Einstein and Sasakian manifolds see [22]). The blow-up of $X_{0}$ at the origin gives $X$, the total space of $K_{M}$, which has a trivial canonical bundle. By the above theorem the total space of $K_{M}$ admits a Ricci-flat metric in every Kähler class.

The set of all Kähler classes forms a cone called the "Kähler cone". In other words, by the Kähler cone of a manifold $M$ we will denote the image of the cone of Kähler metrics in $H^{2}(M, \mathbb{R})$. Linear coordinates in this cone will be called "Kähler moduli". The Kähler cone of the total space of the canonical bundle $K_{\mathscr{F}}$ over the flag manifold is the same as that of the underlying flag manifold. Indeed, one can show that if $k_{0}$ and $r_{0}$ are respectively the Kähler potential and the Ricci-potential of $\mathscr{F}$, then $k=k_{0}+|u|^{2} e^{r_{0}}$ is the Kähler potential of $K_{\mathscr{F}}$, which restricts to $k_{0}$ on the zero section $(u=0)$. This construction essentially dates back to the work of Calabi [12]. The Kähler moduli of the flag manifold, in turn, can be easily characterized geometrically:

- As parameters defining an adjoint orbit, in which case the Kähler form is the Kirillov-Kostant form on this orbit (see [23] for a review).

- As Fayet-Iliopoulos parameters related to the gauged linear $\sigma$-model representations for flag manifolds [24,25].

- The most general $S U(n)$-invariant Kähler metric can be directly constructed using the so-called quasi-potentials [26,27] that also featured in the physics literature in [28]. We will adopt this strategy throughout the paper.

As a result, for the $s$-step flag manifold (i.e. for the flags of type (1.1)) there are $s-1$ real moduli. Therefore Calabi's ansatz does not capture the full moduli space of Ricciflat metrics on the total space. This was taken into account in [29], where a full family of Ricci-flat metrics on the total space of $K_{\mathscr{F}}$ was constructed, using a generalization of Calabi's ansatz. In fact, this generalization is analogous to the one that arose in the 
work [4] on the conifold and was also considered in [7]. The general theory, when such a generalized Calabi's ansatz may be used to construct solutions of the Kähler-Einstein equations, was developed in [30]. The problem of continuation of a given Kähler metric on a manifold to a Ricci-flat metric on the canonical bundle over this manifold was considered in [31].

Another interesting analogy may be observed, if one adds to the above the work [5], where essentially the Ricci-flat Kähler metric on the total space of $K_{\mathbb{C P}^{1} \times \mathbb{C P}^{1}}$ was constructed. As required by the Calabi-Yau theorem, this metric has two Kähler moduli (since $H^{2}\left(\mathbb{C P}^{1} \times \mathbb{C P}^{1}, \mathbb{R}\right) \simeq \mathbb{R}^{2}$ ), which geometrically correspond to the radii of the two spheres representing the 'zero section'. As one of the spheres shrinks (i.e., as we approach the boundary of the Kähler cone in a particular way), one gets a metric on a $\mathbb{C}^{2} / \mathbb{Z}_{2}$ bundle over $\mathbb{C P}^{1}$. Such orbifold bundles were investigated in a more general context in [32]. Removing the orbifold singularity at the zero section, one obtains the total space of the vector bundle $\mathcal{O}(-1) \oplus \mathcal{O}(-1)$ over $\mathbb{C P}^{1}[4]$ (the so-called 'conifold').

1.3. Total spaces of bundles over flag manifolds. In the present paper we pursue a suitable generalization of this procedure to flag manifolds $\mathscr{F}_{n_{1}, \ldots, n_{s}}$. In this case, in place of $\mathbb{C P}^{1} \times \mathbb{C P}^{1}$, we start with the manifold $\mathscr{F}_{n_{1}, \ldots, n_{s}} \times \mathbb{C P}^{q-1}$, and construct a Ricci-flat metric on

$$
X_{K}:=\text { the total space of } K_{\mathscr{F} \times \mathbb{C P}^{q-1}}
$$

In fact, alternatively one may view the manifold $\mathscr{F}_{n_{1}, \ldots, n_{s}} \times \mathbb{C P}^{q-1}$ as a flag manifold of a semi-simple group $S U(n) \times S U(q)$, allowing us to identify these metrics with a special case of the metrics constructed in [29]. We then take the limit, when the volume of $\mathbb{C P}^{q-1}$ vanishes, remove a $\mathbb{C}^{q} / \mathbb{Z}_{q}$-orbifold singularity and show that in the special case when the line bundle $K_{\mathscr{F}}^{1 / q}$ is well-defined (i.e., when $q$ divides $\mathbf{c}_{1}\left(\mathscr{F}_{n_{1}, \ldots, n_{s}}\right)$ ), the resulting manifold is

$$
X_{V}:=\text { the total space of } \frac{K_{\mathscr{F}}^{1 / q} \oplus \cdots \oplus K_{\mathscr{F}}^{1 / q}}{q \text { times }}
$$

over $\mathscr{F}_{n_{1}, \ldots, n_{s}}$. The latter rank- $q$ vector bundle will be denoted by $V$. The logic just described is summarized in Fig. 1.

To formulate our result more precisely, we recall the expression for the first Chern class of the flag manifold:

$$
\mathbf{c}_{1}\left(\mathscr{F}_{n_{1}, \ldots, n_{s}}\right)=-\sum_{k=1}^{s-1}\left(n_{k}+n_{k+1}\right) \mathbf{c}_{1}\left(U_{k}\right),
$$

where $U_{k}$ are pullbacks of tautological bundles over $\operatorname{Gr}\left(m_{k}, n\right)$ w.r.t. the forgetful projections

$$
\pi_{k}: \mathscr{F}_{n_{1}, \ldots, n_{s}} \rightarrow \operatorname{Gr}\left(m_{k}, n\right) .
$$

We recall the derivation of this formula in Sect. 3.1. 


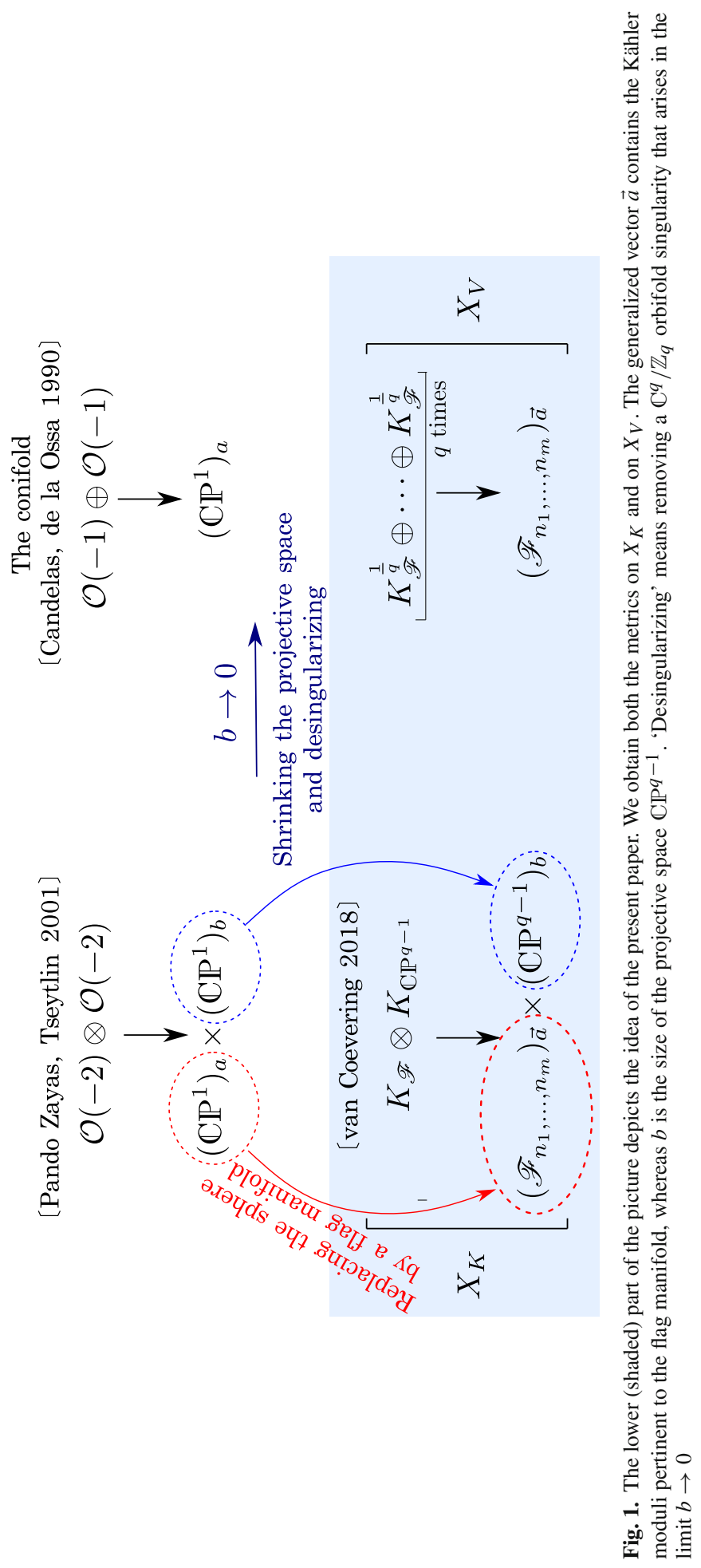


1.4. Explicit Kähler metrics on flag manifolds. In order to formulate our main result, it is useful to recall how the Kähler potential of the most general $S U(n)$-invariant Kähler metric on the flag manifold (1.3) is constructed. To this end consider the matrix

$$
W=\left(w_{1}, \ldots, w_{n}\right) \in G l(n ; \mathbb{C}),
$$

where each $w_{i}$ is a column vector. We also define an $n \times m_{k}$-matrix $Z_{k} \in \operatorname{Hom}\left(\mathbb{C}^{m_{k}}, \mathbb{C}^{n}\right)$ of rank $m_{k}$

$$
Z_{k}=\left(w_{1}, \ldots, w_{m_{k}}\right), \text { where } m_{k}=\sum_{l=1}^{k} n_{l},
$$

and introduce the function

$$
t_{k}=\operatorname{det}\left(Z_{k}^{\dagger} Z_{k}\right)
$$

One can check that $\log \left(t_{k}\right)$ is the Kähler potential for the $\pi$-normalized canonical metric ${ }^{1}$ on the Grassmannian $G r\left(m_{k}, n\right)$. The potential of an arbitrary $S U(n)$ invariant Kähler metric on the flag manifold $[26,27]$ may then be written as

$$
\mathscr{K}_{\mathscr{F}}=\sum_{k=1}^{s-1} \gamma_{k} \log \left(t_{k}\right), \quad \gamma_{k}>0
$$

Additionally we will use (a multiple of) the standard Fubini-Study metric on $\mathbb{C P}^{q-1}$, with Kähler potential

$$
\mathscr{K}_{\mathbb{C P}^{q-1}}=\gamma_{0} \log \left(t_{0}\right):=\gamma_{0} \log \left(\sum_{i=1}^{q}\left|z_{i}\right|^{2}\right), \quad \gamma_{0}>0 .
$$

Cohomology classes of the metrics (1.11) exhaust the Kähler cone of the flag manifold. To see this, we introduce the two-dimensional Schubert classes $\overline{\mathcal{C}}_{i}$, which are closures of the cells ( $E_{i j}$ is the matrix with a single non-zero element, equal to 1 , in the $i j$-th position)

$$
\mathcal{C}_{i}=\left\{W \in G L(n, \mathbb{C}) / P_{m_{1}, \ldots, m_{s}}: W=\mathbf{1}_{n}+z E_{m_{i}+1 m_{i}}\right\} \quad i=1, \ldots, s-1 .
$$

In this case all $\overline{\mathcal{C}}_{i}$ 's are copies of $\mathbb{C P}^{1}$, and $z$ in the above formula is the stereographic coordinate. Since these are holomorphic cycles, the integral of any Kähler form $\omega$ (not necessarily $S U(N)$-invariant) over such a cycle is proportional to its volume and hence is positive: $\int_{\overline{\mathcal{C}}_{i}} \omega>0(i=1, \ldots, s-1)$. On the other hand, because $\overline{\mathcal{C}}_{i}$ generate the homology group $H_{2}(\mathscr{F}, \mathbb{R})$, these integrals determine the Kähler class of $\omega$. To see if there any restrictions on the volumes, we evaluate the integrals for the metrics (1.11), in which case $\int \omega \sim \gamma_{i}$. Therefore the volumes may be chosen independently. $\overline{\mathcal{C}}_{i}$

1 The same normalization as that of the Fubini-Study metric on $\mathbb{C P}^{n-1}$, i.e. the volume of a holomorphic 2-sphere generating $\mathrm{H}_{2}\left(\mathrm{Gr}\left(m_{k}, n\right), \mathbb{Z}\right)$ is $\pi$. 
1.5. Ricci-flat metrics on vector bundles. We prove the following statement:

Proposition. There exists a complete Ricci-flat Kähler metric on $X_{K}$ in each Kähler class. If there exists a $q \in \mathbb{N}$ such that $q \mid\left(n_{k}+n_{k+1}\right)(k=1 \ldots s-1)$, then there exists a complete Ricci-flat Kähler metric on $X_{V}$ in each Kähler class. In both cases the line element on the total space is of the form

$$
\begin{aligned}
& d s^{2}=\sum_{k=0}^{s-1} \gamma_{k}(\mu) \partial \bar{\partial} \log t_{k}+\frac{Q(\mu)}{4} d \mu^{2}+\frac{1}{Q(\mu)}(d \phi+\operatorname{Im}(A))^{2}, \\
& \text { where } \quad\left(c_{k}:=n_{k}+n_{k+1}\right) \\
& \gamma_{0}(\mu)=q(\alpha+\mu), \quad \gamma_{k}(\mu)=c_{k}\left(a_{k}+\mu\right) \quad(k \geq 1), \\
& Q(\mu)=\frac{F^{\prime}(\mu)}{F(\mu)}, \quad F(\mu)=\int_{C}^{\mu} d \mu^{\prime}\left(\alpha+\mu^{\prime}\right)^{q-1} \prod_{1 \leq i<j \leq s}\left(\sum_{k=i}^{j-1} c_{k}\left(a_{k}+\mu^{\prime}\right)\right)^{n_{i} n_{j}}
\end{aligned}
$$

In the above formula $A$ is the holomorphic connection of the canonical bundle over $\mathscr{F} \times \mathbb{C P}^{q-1}$ and is explicitly given by

$$
A:=\partial\left(q \log t_{0}+\sum_{k=1}^{s-1} c_{k} \log t_{k}\right) .
$$

The choice of complex structure $\mathscr{J}$ on the flag manifold was discussed in Sect. 1.1 and may also be inferred from Sect. 1.4. The action of $\mathscr{J}$ on $(d \mu, d \phi)$ is given by $\mathscr{J}\left(\frac{1}{2} Q(\mu) d \mu\right)=d \phi+\operatorname{Im}(A)$.

The real constants $\left(a_{k}, \alpha\right)$ satisfy certain inequalities (and are otherwise arbitrary) and determine the Kähler class of the metric:

If $\alpha+C>0, a_{k}+C>0$ for all $k$, and the angular variable $\phi$ takes values in $[0,2 \pi]$ (1.14)-(1.15) describe a metric on $X_{K}$ for all Kähler classes. In this case $\phi$ is the phase of the complex coordinate in the fiber.

If $a_{k}-\alpha>0$ for all $k, C=-\alpha$, and $\phi \in[0,2 \pi q]$ (1.14)-(1.15) describe a metric on $X_{V}$ for all Kähler classes. Viewing the fiber without origin $\mathbb{C}^{q} \backslash\{0\}$ as the total space of $a \mathbb{C}^{*}$ bundle over $\mathbb{C P}^{q-1}$ and parameterizing $\mathbb{C}^{*}$ by $x_{0}$, we have $\frac{\phi}{q}=\arg \left(x_{0}\right)$.

In both cases $\mu$ is the moment map associated to the $\mathfrak{u}_{1}$ action of the holomorphic isometry $\phi \rightarrow \phi+$ const.

The metrics so constructed are asymptotic to the Riemannian cone over the SasakiEinstein $U(1)$ bundles over $\mathscr{F}_{n_{1}, \ldots, n_{s}} \times \mathbb{C P}^{q-1}$. For $X_{K}$ and $X_{V}$ these are the unit vector bundles of $K_{\mathscr{F}_{n_{1}, \ldots, n_{S}}} \times \mathbb{C P}^{q-1}$ and its q th root respectively.

Comment 1 We note that the line element (1.14) has the form of Pedersen and Poon [33]. Comment 2 The metric (1.14) is of cohomogeneity one w.r.t. the action of the symmetry group $S U(n) \times S U(q)$. For the manifolds $X_{K}$ and $X_{V}$ the metrics restrict to $S U(n) \times$ $S U(q)$-invariant and $S U(n)$-invariant metrics on the base respectively. We note that the general theory of cohomogeneity-one Kähler manifolds was developed in [34-36].

Comment 3 After the first version of the present paper appeared, the sequel [37] to [36] came out, where the authors construct Ricci-flat metrics on vector bundles over flag manifolds. Our construction for $X_{V}$ may be viewed as a particular case, when the typical orbit of the symmetry group is a $U(1)$-bundle over the reducible flag manifold $\mathscr{F}_{n_{1}, \ldots, n_{m}} \times$ 
$\mathbb{C P}^{q-1}$. In the language of [37], this means starting from a disconnected (painted) Dynkin diagram of $\mathfrak{s u}_{n} \oplus \mathfrak{s u}$, where all the nodes of $\mathfrak{s u}_{q}$ are white ('a white $A_{q-1}$ string').

The structure of the paper is as follows. In Sect. 2 we recall, how the Ricci-flat Kähler metric on $K_{\mathrm{CP}^{1} \times \mathbb{C P}^{1}}$ is constructed, using a generalization of Calabi's ansatz. In Sect. 3 we pass over to flag manifolds, starting in Sect. 3.1 by explaining the expression for the first Chern class of a flag manifold and constructing the Kähler-Einstein metric in explicit form. Using the Kähler-Einstein metric, we construct in Sect. 3.2 a generalization of Calabi's ansatz, which allows obtaining the Ricci-flat metric in every Kähler class. This generalized ansatz leads to an ODE, which is then solved in Sect. 3.2. In the same section the topology of the manifold (the behavior near the zero section, as well as at infinity) is also analyzed. The "Appendix" is dedicated to the calculation of the determinant of the Hermitian metric, which is used in writing out the Ricci-flatness equation.

\section{Conifold and Canonical Bundle Over $\mathbb{C P}^{1} \times \mathbb{C P}^{1}$}

The ansatz of Calabi may be succinctly formulated as the requirement that the Kähler potential $\mathscr{K}$ on the total space assumes the form

$$
\mathscr{K}=\mathscr{K}\left(|u|^{2} e^{\widehat{K}}\right),
$$

where $\widehat{K}$ is the Kähler potential of the Kähler-Einstein metric on the underlying manifold $\mathcal{M}_{K E}$ and $u$ is the coordinate in the fiber. Throughout the paper we will assume that the Kähler-Einstein metric $g_{K E}$ on the base is normalized so that

$$
\text { Ric }=g_{K E} .
$$

In this section as our principal example we will take $\mathcal{M}_{K E}=\mathbb{C P}^{1} \times \mathbb{C P}^{1}$. Note that this manifold has two Kähler moduli (the sizes of the two spheres), so this is the simplest instance of the situation described in the introduction, namely the Calabi-Yau theorem requires the existence of two parameters in the metric on $Y_{K}:=$ the total space of $K_{\mathbb{C P}^{1} \times \mathbb{C P}^{1}}$. Calabi's ansatz (2.1) fails to capture the full moduli space, since the Kähler-Einstein condition on $\mathbb{C P}^{1} \times \mathbb{C P}^{1}$ requires that the radii of the two $\mathbb{C P}^{1}$ 's be equal.

Interestingly, Calabi's ansatz corresponds to a special point in the moduli space of metrics, namely the corresponding Kähler form $\left[\omega_{\text {Calabi }}\right] \in H_{c}^{2}\left(Y_{K}, \mathbb{R}\right)$ lies in the compactly supported cohomology. This is characterized by a faster decay to the asymptotic form at infinity. For a more detailed discussion of this see [20,21,38].

Denoting the inhomogeneous coordinates on the two spheres by $z$ and $w$, one introduces the following generalization of Calabi's ansatz (after a simple change of variables):

$$
\begin{aligned}
& \mathscr{K}=a_{1} K_{1}+a_{2} K_{2}+\mathscr{K}_{0}\left(\frac{|u|^{2} e^{K_{1}+K_{2}}}{:=x}\right), \\
& K_{1}=2 \log \left(\left|z_{1}\right|^{2}+\left|z_{2}\right|^{2}\right), \quad K_{2}=2 \log \left(\left|w_{1}\right|+\left|w_{2}\right|^{2}\right) .
\end{aligned}
$$

Clearly $K_{1}$ and $K_{2}$ are the Kähler potentials of the two spheres, and $K_{1}+K_{2}$ is the Kähler potential of the Einstein metric on $\mathbb{C P}^{1} \times \mathbb{C P}^{1}$. Setting $a_{1}=a_{2}=0$ would yield precisely the ansatz of Calabi. 
Now, we are dealing with a toric variety, the $U(1)^{3}$ holomorphic isometries being given by the rotations

$$
\left(z_{1}, z_{2}\right) \rightarrow\left(e^{i \alpha_{1}} z_{1}, e^{-i \alpha_{1}} z_{2}\right), \quad\left(w_{1}, w_{2}\right) \rightarrow\left(e^{i \alpha_{2}} w_{1}, e^{-i \alpha_{2}} w_{2}\right), \quad u \rightarrow e^{i \beta} u .
$$

In such cases it is useful to pass to the moment map variables. The moment map is defined as the derivative $\nabla \mathscr{J} v \mathscr{K}$, where $\mathscr{J}$ is the complex structure, and $v$ is the vector field corresponding to the holomorphic isometry. In practical terms, this is tantamount to replacing $\left|z_{1}\right|^{2}+\left|z_{2}\right|^{2} \rightarrow e^{s_{1}}+e^{-s_{1}},\left|w_{1}\right|^{2}+\left|w_{2}\right|^{2} \rightarrow e^{s_{2}}+e^{-s_{2}},|u|^{2} \rightarrow e^{t}$ in the Kähler potential, and differentiating it w.r.t. $s_{i}$ and $t$ :

$$
\mu=\frac{\partial \mathscr{K}}{\partial t}=x \mathscr{K}_{0}^{\prime}(x), \quad v_{i}=\frac{\partial \mathscr{K}}{\partial s_{i}}=2 \operatorname{th}\left(s_{i}\right)\left(a_{i}+\mu\right) .
$$

One also computes the so-called symplectic potential $\mathscr{H}$, which is the Legendre transform of the Kähler potential $\mathscr{K}$ w.r.t. the variables $t, s_{1}, s_{2}$ (for details of this procedure see [39, Sec. 2]):

$$
\mathscr{H}\left(\mu, v_{1}, v_{2}\right)=t \mu+s_{1} v_{1}+s_{2} v_{2}-\mathscr{K} .
$$

The nice feature of this potential is that the domain, on which it is defined, is precisely the moment polytope of the manifold. Clearly, since in our case the manifold is non-compact, the polytope is also unbounded.

Up to inessential linear terms in $\mu$, the symplectic potential reads:

$$
\begin{array}{r}
\mathscr{H}=H(\mu)+\sum_{i=1}^{2} \ell_{i}^{+} \log \ell_{i}^{+}+\sum_{i=1}^{2} \ell_{i}^{-} \log \ell_{i}^{-}-2 \sum_{i=1}^{2}\left(\mu+a_{i}\right) \log \left(\mu+a_{i}\right), \\
\text { where } \quad H(\mu)=\mu \log x-\mathscr{K}_{0}, \quad \ell_{i}^{ \pm}=\mu+a_{i} \pm \frac{1}{2} v_{i} .
\end{array}
$$

Note that the $l \log (l)$ structures appearing in the symplectic potential are typical for Kähler toric geometry [40]. Now, the function $H(\mu)$ is determined from the Ricciflatness equation, which in this case reads

$$
2\left(\mu+a_{1}\right)\left(\mu+a_{2}\right)=\frac{d}{d \mu}\left(e^{H_{\mu}}\right) .
$$

The solution is $H=\sum_{i=0}^{2}\left(\mu-\mu_{i}\right)\left(\log \left(\mu-\mu_{i}\right)-1\right)$, where $\mu_{i}$ are the three roots of the polynomial $F_{0}(\mu)=\int_{\mu_{0}}^{\mu} d \mu^{\prime} 3\left(\mu^{\prime}+a_{1}\right)\left(\mu^{\prime}+a_{2}\right)$.

Next we write down the explicit expression for the line element derived from the Kähler potential (2.3), using the dual variable $\mu$ :

$$
\begin{aligned}
d s^{2} & =\left(\mu+a_{1}\right)\left(d s^{2}\right)_{\mathbb{C P}_{z}^{1}}+\left(\mu+a_{2}\right)\left(d s^{2}\right)_{\mathbb{C P}_{w}^{1}}+\frac{Q_{0}(\mu)}{4} d \mu^{2}+\frac{1}{Q_{0}(\mu)}(d \phi+\operatorname{Im}(A))^{2}, \\
Q_{0}(\mu) & =H_{\mu \mu}=\frac{F_{0}^{\prime}(\mu)}{F_{0}(\mu)}, \quad F_{0}(\mu)=\int_{\mu_{0}}^{\mu} d \mu^{\prime} 3\left(\mu^{\prime}+a_{1}\right)\left(\mu^{\prime}+a_{2}\right), \\
\left(d s^{2}\right)_{\mathbb{C P}_{z}^{1}} & =\frac{2 d z d \bar{z}}{\left(1+|z|^{2}\right)^{2}}, \quad\left(d s^{2}\right)_{\mathbb{C P}_{w}^{1}}=\frac{2 d w d \bar{w}}{\left(1+|w|^{2}\right)^{2}},
\end{aligned}
$$




$$
A=2\left(\frac{\bar{z} d z}{1+|z|^{2}}+\frac{\bar{w} d w}{1+|w|^{2}}\right)
$$

Here $A$ is the holomorphic connection on the canonical bundle $K_{\mathbb{C P}^{1} \times \mathbb{C P}^{1}}$. Nonnegativity of the metric implies $\mu+a_{1} \geq 0, \mu+a_{2} \geq 0, H_{\mu \mu}=\frac{F_{0}^{\prime}(\mu)}{F_{0}(\mu)} \geq 0$. The latter requirement is equivalent to $F_{0}(\mu) \geq 0$. Note that $F_{0}\left(\mu_{0}\right)=0$ and $F_{0}(\mu)>0$ for $\mu>\mu_{0}$, due to the first two inequalities. Completeness requires that the range of $\mu$ is $\mu \in\left[\mu_{0}, \infty\right)$. In fact, there are two distinct possibilities:

- $\mu_{0}>\max \left(-a_{1},-a_{2}\right)$. This was considered by Pando Zayas and Tseytlin [5].

- $\mu_{0}=\max \left(-a_{1},-a_{2}\right)$. This was considered in the early work of Candelas and de la Ossa [4].

In the first case, one has $H_{\mu \mu}=\frac{1}{\mu-\mu_{0}}+\cdots$ as $\mu \rightarrow \mu_{0}$. The change of variables $r=\left(\mu-\mu_{0}\right)^{1 / 2}$ brings the metric to the asymptotic form

$$
\left(d s^{2}\right)_{\mu \rightarrow \mu_{0}}=\left(\mu_{0}+a_{1}\right)\left(d s^{2}\right)_{\mathbb{C P}_{z}^{1}}+\left(\mu_{0}+a_{2}\right)\left(d s^{2}\right)_{\mathbb{C P}_{w}^{1}}+d r^{2}+r^{2}(d \phi+\operatorname{Im}(A))^{2} .
$$

The absence of singularity at $r=0$ requires that the angle $\phi$ has the range $\phi \in[0,2 \pi]$. Then (2.10) shows that one has the canonical bundle, with connection $\operatorname{Im}(A)$, over $\mathbb{C P}^{1} \times \mathbb{C P}^{1}$, the two spheres having radii squared $\mu_{0}+a_{1}$ and $\mu_{0}+a_{2}$. Varying $\mu_{0}$ leads to changing the Kähler class of the metric, and all allowed classes (corresponding to the non-vanishing sizes of the $\mathbb{C P}^{1}$ 's) can be achieved in this way.

In the second case, let us assume that $\max \left(-a_{1},-a_{2}\right)=-a_{1} . F_{0}(\mu)$ now has a second-order zero at $\mu=\mu_{0}$, so that $H_{\mu \mu}=\frac{2}{\mu-\mu_{0}}+\cdots$ Introducing the variable $r=\left(2\left(\mu-\mu_{0}\right)\right)^{1 / 2}$, in the vicinity of $\mu=\mu_{0}$ we can bring the metric to the form

$$
\left(d s^{2}\right)_{\mu \rightarrow \mu_{0}}=\left(a_{2}-a_{1}\right)\left(d s^{2}\right)_{\mathbb{C P}_{z}^{1}}+d r^{2}+r^{2}\left(\frac{d w d \bar{w}}{\left(1+|w|^{2}\right)^{2}}+\left(d \tilde{\phi}+\frac{1}{2} \operatorname{Im}(A)\right)^{2}\right) .
$$

Here $\tilde{\phi}=\frac{\phi}{2}$. If one sets $z=$ const., the expression in round brackets is precisely the round metric on $S^{3}$, provided one takes the periodicity of the angle $\tilde{\phi}$ to be $\tilde{\phi} \in[0,2 \pi]$. In this case the conical part $d r^{2}+r^{2}(\ldots)$ of the metric above describes the space $\mathbb{R}^{4} \simeq \mathbb{C}^{2}$. (Keeping instead the periodicity $\phi \in[0,2 \pi]$ would lead to a $\mathbb{C}^{2} / \mathbb{Z}_{2}$ orbifold singularity at the zero section, $r=0$.) Taking into account the stereographic $z$-variable of the remaining sphere $\mathbb{C P}^{1}$, one can show that the metric (2.11) describes the vicinity of the zero section in the $\mathcal{O}(-1) \oplus \mathcal{O}(-1)$ bundle over $\mathbb{C P}^{1}$ (the so-called 'conifold') [4].

Since the varieties we have considered are toric, it is instructive to construct the respective toric polytopes. The polytopes corresponding to the Pando Zayas-Tseytlin solution and the Candelas-de la Ossa solution are schematically presented in Figs. 2 and 3 , respectively. The sections of the moment polytope in the $(\mu, v)$ plane are shown to the left of the full three-dimensional polytope.

\section{Flag Manifolds}

We will start by recalling the explicit form of the Kähler-Einstein metric on the flag manifold, which is an essential ingredient in Calabi's ansatz. We then use a suitable generalization of this ansatz to construct the metric on $X_{K}$-the total space of the canonical bundle over $\mathscr{F}_{n_{1}, \ldots, n_{s}} \times \mathbb{C P}^{q-1}$, and on $X_{V}$-the total space of the vector 

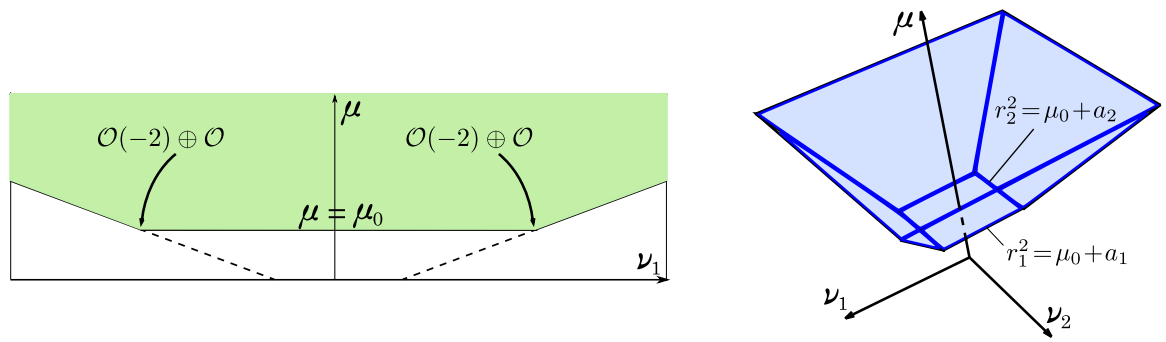

Fig. 2. The moment polytope for the cone over $\mathbb{C P}^{1} \times \mathbb{C P}^{1}$ (right) and its section in the $\left(\mu, v_{1}\right)$ plane (left). The radii of the two spheres representing the zero section are denoted $r_{1}, r_{2}$
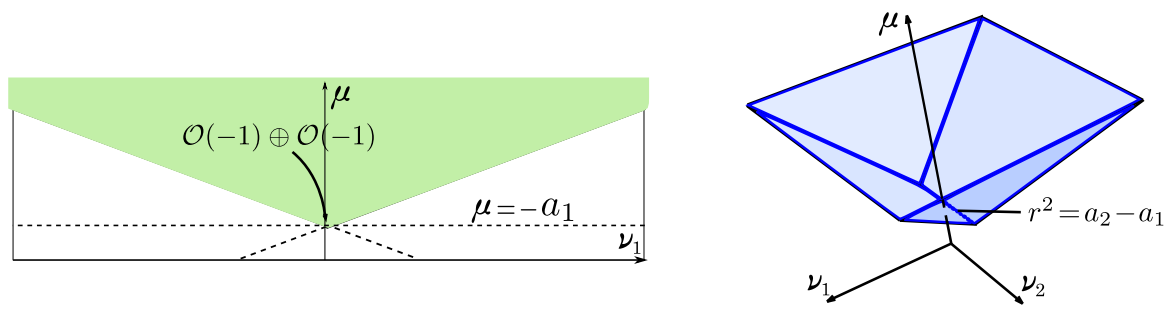

Fig. 3. The moment polytope for the total space of $\mathcal{O}(-1) \oplus \mathcal{O}(-1)$ vector bundle over $\mathbb{C P}^{1}$ (right) and its section in the $\left(\mu, v_{1}\right)$ plane (left). The radius of the sphere representing the zero section is denoted $r$

bundle $V:=\underbrace{K_{\mathscr{F}}^{1 / q} \oplus \cdot \oplus K_{\mathscr{F}}^{1 / q}}_{q \text { times }}$ (the latter in the case when the $q$-th root of $K_{\mathscr{F}}$ makes sense).

3.1. The canonical bundle. On $\mathscr{F}_{n_{1}, \ldots, n_{s}}$ we can consider the vector bundles $\xi_{j}$ and $U_{j}$ $(j=1, \ldots, s)$ where the fiber of $\xi_{j}$ over the point

$$
0 \subset L_{1} \subset \cdots \subset L_{s-1} \subset L_{s}=\mathbb{C}^{n}
$$

is given by $L_{j} / L_{j-1}$, and the fiber of $U_{j}$ is $L_{j}$ ( $U_{j}$ are the tautological bundles). Here, as before, $L_{k} \cong \mathbb{C}^{m_{k}}$. As is well-known [41]

$$
T \mathscr{F}_{n_{1}, \ldots, n_{s}}=\bigoplus_{1 \leq i<j \leq s} \xi_{i}^{*} \otimes \xi_{j}
$$

We will be interested in the explicit expression for the first Chern class of the flag manifold:

$$
\mathbf{c}_{1}\left(\mathscr{F}_{n_{1}, \ldots, n_{s}}\right)=\sum_{i<j}\left(-n_{j} \mathbf{c}_{1}\left(\xi_{i}\right)+n_{i} \mathbf{c}_{1}\left(\xi_{j}\right)\right) .
$$

Rewriting in terms of $U_{j}$ gives

$$
\mathbf{c}_{1}\left(\mathscr{F}_{n_{1}, \ldots, n_{s}}\right)=-\sum_{k=1}^{s-1}\left(n_{k}+n_{k+1}\right) \mathbf{c}_{1}\left(U_{k}\right) .
$$


Now, suppose there is a positive integer $q$ that divides $\left(n_{k}+n_{k+1}\right)$ for all $k$. Then, since $\operatorname{Pic}(\mathscr{F}) \simeq H^{2}(\mathscr{F}, \mathbb{Z})$ [42, Prop. 2.1.2], [43], there is a line bundle $K_{\mathscr{F}}^{1 / q}$, where $K_{\mathscr{F}}$ is the canonical bundle of $\mathscr{F}_{n_{1}, \ldots, n_{s}}$.

Furthermore since $\mathscr{F}_{n_{1}, \ldots, n_{s}}$ is Kähler we have that the Ricci-form represents $\mathbf{c}_{1}\left(\mathscr{F}_{n_{1}, \ldots, n_{s}}\right)$. Let $\left(u_{1}, \ldots, u_{n}\right) \in U(n)$, where $u_{i}$ are column vectors. Then

$$
\mathbf{c}_{1}\left(U_{k}\right)=\left[\frac{1}{2 \pi i} \sum_{i=1}^{m_{k}} \sum_{j=m_{k}+1}^{n} J_{i j} \wedge J_{j i}\right]
$$

with $J_{i j}=\sum_{m} \bar{u}_{j m} d u_{i m}$. Therefore the line element of the Kähler-Einstein metric on $\mathscr{F}_{n_{1}, \ldots, n_{s}}$, satisfying (2.2), must take the form

$$
d s^{2}=\sum_{k=1}^{s-1}\left(n_{k}+n_{k+1}\right) \sum_{i=1}^{m_{k}} \sum_{j=m_{k}+1}^{n}\left|J_{i j}\right|^{2}
$$

Note that Kähler-Einstein metric on flag manifolds were first discussed in [15]. The Kähler potential corresponding to this metric has the form (1.11) with $\gamma_{k}=n_{k}+n_{k+1}:=c_{k}$.

3.2. Generalized Calabi Ansatz for all Kähler classes. From now on we assume there is a positive integer $q$ that divides $c_{k}:=n_{k}+n_{k+1}$ for all $k=1, \ldots, s-1$. Besides, we define the vector $\vec{z}=\left(z_{1}, \ldots, z_{q-1}\right) \in \mathbb{C}^{q-1}$.

As a candidate for a Kähler potential on $X_{K}$, we define

$$
\mathscr{K}=\alpha q \log \left(1+|\vec{z}|^{2}\right)+\sum_{k=1}^{s-1} a_{k} c_{k} \log t_{k}+\mathscr{K}_{0}(\underbrace{\left.|u|^{2}\left(1+|\vec{z}|^{2}\right)^{q} \Pi_{k} t_{k}^{c_{k}}\right)}_{=: e^{t}} .
$$

From the discussion in the previous section it follows that $\widehat{K}=q \log \left(1+|\vec{z}|^{2}\right)+$ $\sum_{k=1}^{s-1} c_{k} \log \left(t_{k}\right)$ is the Kähler potential of the Kähler-Einstein metric on $\mathscr{F}_{n_{1}, \ldots, n_{s}} \times \mathbb{C P}^{q-1}$, and therefore the last term in (3.7) is the expression familiar from Calabi's ansatz (2.1).

In (3.7) $\left(\alpha, a_{k}\right)$ are the parameters (Kähler moduli), akin to $a_{1}, a_{2}$ from (2.3). Their range will be determined later. If we work on $X_{K}$, the components $z_{i}$ of $\vec{z}$ are local coordinates on $\mathbb{C P}^{q-1}$ and $u$ is a holomorphic coordinate on the fiber. If we work on $X_{V},\left(u, z_{i}\right)$ are local coordinates on the fiber.

Notice that $\mathscr{K}_{0}$ depends only on a single variable $t$. We will write $\mathscr{K}_{0}^{\prime}$ and $\mathscr{K}_{0}^{\prime \prime}$ for its first and second derivatives w.r.t $t$. In fact, instead of dealing with the function $\mathscr{K}_{0}$, just like in Sect. 2 it is convenient to perform a Legendre transform

$$
H=\mu t-\mathscr{K}_{0},
$$

whence

$$
\mathscr{K}_{0}^{\prime}=\mu, \quad \mathscr{K}_{0}^{\prime \prime}=\frac{1}{H_{\mu \mu}} .
$$

The meaning of $\mu$ is that it is the moment map for the $U(1)$-action $u \rightarrow e^{i \alpha} u$. 
The line element then is given by

$$
d s^{2}=q(\alpha+\mu) d s_{F S}^{2}+\sum_{k=1}^{s-1} c_{k}\left(a_{k}+\mu\right) \pi_{k}^{*}\left(d s_{k}^{2}\right)+\frac{1}{|u|^{2} H_{\mu \mu}}|d u+u A|^{2},
$$

where $d s_{F S}^{2}$ has the form of a Fubini-Study metric on $\mathbb{C P}^{q-1}$ and

$$
\begin{aligned}
\pi_{k}^{*}\left(d s_{k}^{2}\right) & =\left(\partial_{i} \bar{\partial}_{j} \log t_{k}\right) d y^{i} d \bar{y}^{j} \\
A & =\sum_{i, k} c_{k} \partial_{i} \log t_{k} d y^{i}+q \partial_{i} \log \left(1+|\vec{z}|^{2}\right) d z^{i} .
\end{aligned}
$$

Note that $y^{i}$ are the complex coordinates on the flag manifold, and are abbreviations for $w_{l k}$ (the components of the vectors $w_{l}$ ).

The expression (3.10) may be brought to the form (1.14) used in the statement of the Proposition, if we introduce the angular variable $\phi=\arg (u)$. Then, using the definition of $t$ from (3.7), we obtain

$$
\log u=\frac{t}{2}+i \phi-\frac{q}{2} \log \left(1+|\vec{z}|^{2}\right)-\frac{c_{k}}{2} \sum_{k} \log t_{k} .
$$

Since $t=H_{\mu}$, we arrive at

$$
\frac{d u}{u}+A=d \log u+A=\frac{H_{\mu \mu} d \mu}{2}+i D \phi
$$

with $D \phi=d \phi+\operatorname{Im}(A)$ as covariant derivative. As the 1.h.s. of the above equality is a one-form of type $(1,0)$, on which the complex structure $\mathscr{J}$ acts by multiplication by $-i$, we get

$$
\mathscr{J}\left(\frac{H_{\mu \mu} d \mu}{2}\right)=D \phi .
$$

The $u$-dependent part of the metric (3.10) is then

$$
\frac{1}{H_{\mu \mu}}\left|\frac{d u}{u}+A\right|^{2}=H_{\mu \mu} \frac{d \mu^{2}}{4}+\frac{(D \phi)^{2}}{H_{\mu \mu}} .
$$

Note that the function $Q$ mentioned in the proposition thus satisfies $Q(\mu)=H_{\mu \mu}$. We denote by $g$ the Hermitian (Kähler) metric corresponding to (3.10). The Riccitensor is given by $R_{i} \bar{j}=-\partial_{i} \bar{\partial}_{j} \log \operatorname{det} g$, where $\partial_{i}$ are derivatives w.r.t. the holomorphic coordinates. Therefore Ricci-flatness is satisfied if

$$
\operatorname{det} g \sim \kappa \bar{\kappa}
$$

for a holomorphic function $\kappa$. It will be shown in Appendix B that

$$
\operatorname{det} g=\left|\kappa\left(\left\{w_{m i}\right\}\right)\right|^{2} \frac{1}{|u|^{2} H_{\mu \mu}} \frac{q^{q-1}(\alpha+\mu)^{q-1}}{\left(1+|\vec{z}|^{2}\right)^{q}} \frac{1}{\prod_{k} t_{k}^{c_{k}}} f(\mu),
$$

where $\kappa$ is a holomorphic function, and

$$
f(\mu)=\prod_{1 \leq i<j \leq s}\left(\sum_{k=i}^{j-1} c_{k}\left(a_{k}+\mu\right)\right)^{n_{i} n_{j}}
$$


Using the definition

$$
e^{t}=|u|^{2}\left(1+|\vec{z}|^{2}\right)^{q} \prod_{k} t_{k}^{c_{k}}
$$

from (3.7), as well as $H_{\mu}=t$, we may satisfy (3.17) by requiring

$$
(\alpha+\mu)^{q-1} f(\mu)=H_{\mu \mu} \exp \left(H_{\mu}\right)=\partial_{\mu} \exp \left(H_{\mu}\right)
$$

3.3. Solution of the Ricci-flatness equation. The ODE (3.21) is solved by

$$
H_{\mu}=\log \left(\int_{C}^{\mu} d \mu^{\prime}\left(\alpha+\mu^{\prime}\right)^{q-1} f\left(\mu^{\prime}\right)\right) .
$$

Therefore

$$
H_{\mu \mu}=\frac{(\alpha+\mu)^{q-1} f(\mu)}{\int_{C}^{\mu} d \mu^{\prime}\left(\alpha+\mu^{\prime}\right)^{q-1} f\left(\mu^{\prime}\right)} .
$$

3.3.1. Behavior at $\infty$ Before analysing the effect of different choices for the integration constant $C$, we observe that

$$
H_{\mu \mu} \rightarrow \frac{N}{\mu} \quad \text { as } \quad \mu \rightarrow \infty .
$$

Here $N=q+\operatorname{dim}_{\mathbb{C}}(\mathscr{F})$. Using (3.10) and (3.16) and making the substitution $\mu=\frac{1}{N} r^{2}$, we find that the line element behaves at infinity as

$$
\left.d s^{2}\right|_{\mu \rightarrow \infty}=d r^{2}+r^{2}\left(\frac{1}{N} d s_{K E}^{2}+\frac{1}{N^{2}}(D \phi)^{2}\right),
$$

where $d s_{K E}^{2}=q d s_{F S}^{2}+\sum_{k=1}^{s-1} c_{k} \pi_{k}^{*}\left(d s_{k}^{2}\right)$ is the Kähler-Einstein metric on $\mathscr{F}_{n_{1}, \ldots, n_{s}} \times$ $\mathbb{C P}^{q-1}$ with proportionality factor 1 . Thus at $\mu \rightarrow \infty$ we obtain a cone over a Sasakian manifold, which is a $U(1)$-bundle over $\mathscr{F}_{n_{1}, \ldots, n_{s}} \times \mathbb{C P}^{q-1}$. As will be demonstrated in the remainder of this section the angle $\phi$ ranges from $[0,2 \pi]$ on $X_{K}$ and from $[0,2 \pi q]$ on $X_{V}$. Therefore the $U(1)$-bundles are the unit-vector bundles of $K_{\mathscr{F}_{n_{1}}, \ldots, n_{S}} \times \mathbb{C P}^{q-1}$ and $K_{\mathscr{F}_{n_{1}, \ldots, n_{s}} \times \mathbb{C P}^{q-1}}^{1 / q}$ respectively.

3.3.2. The metric on $X_{K}$ We now analyze the dependence on the choice of $C$. If $C$ is larger than the largest root of $(\alpha+\mu)^{q-1} f(\mu)$, then $H_{\mu \mu}$ is strictly positive. The only possible issue would be a singularity at the zero section, but since

$$
H_{\mu \mu}=\frac{1}{\mu-C}+O(1), \quad \text { as } \quad \mu \rightarrow C,
$$

the substitution $r^{2}=\mu-C$ implies

$$
\frac{1}{H_{\mu \mu}}\left|\frac{d u}{u}+A\right|^{2}=d r^{2}+r^{2}(D \phi)^{2}+\cdots,
$$


that is to say the line element corresponds to a smooth metric. The complex variable $r e^{i \phi}$ parametrizes $\mathbb{C}$ - the fiber of a line bundle, and the connection $A$ allows to identify this bundle with $K_{\mathscr{F} \times \mathbb{C P}^{q-1}}$. Therefore the underlying manifold is $X_{K}$. The condition

$$
a_{k}+C>0, \quad \alpha+C>0
$$

makes the metric positive-definite. Upon restricting to the zero section $\mu=C$, (3.10) reduces to

$$
d s^{2}=\gamma_{0} d s_{F S}^{2}+\sum \gamma_{k} \pi_{k}^{*}\left(d s_{k}^{2}\right),
$$

with $\gamma_{0}=q(\alpha+C), \gamma_{k}=c_{k}\left(a_{k}+C\right)$. The definition (1.11) of the Kähler cone of the flag manifold, combined with $\gamma_{0}>0$ for $\mathbb{C P}^{q-1}$, implies that all Kähler classes are obtained.

3.3.3. The metric on $X_{V}$ We will now show that for $C=-\alpha$ the line element (3.10) describes a metric on $X_{V}$. Notice that positive-definiteness of the metric (3.35) requires (since $c_{k}>0$ for all $k$ )

$$
a_{k}-\alpha>0 .
$$

It then follows easily from (3.19), (3.23) that the above condition also makes $H_{\mu \mu}$ (and hence the metric (1.14)) positive for $\mu>-\alpha$.

One can visualize the limit $\mu \rightarrow-\alpha$ as sending the volume of $\mathbb{C P}^{q-1}$ to zero and embedding $\mathbb{C} \mathbb{P}^{q-1}$ into the fiber $\mathbb{C}^{q}$. First we notice that

$$
\int_{-\alpha}^{\mu} d \mu^{\prime}\left(\alpha+\mu^{\prime}\right)^{q-1} f\left(\mu^{\prime}\right)=\delta(\alpha+\mu)^{q}(1+O(\alpha+\mu)), \quad \delta=\frac{f(-\alpha)}{q}>0 .
$$

Since $t=H_{\mu}$, (3.22) yields

$$
e^{t}=\delta(\alpha+\mu)^{q}(1+O(\alpha+\mu))
$$

Therefore to leading order in $\alpha+\mu$

$$
\begin{aligned}
\mathscr{K}_{0} & =\mu H_{\mu}-H \\
& =\mu \log \left(\delta(\alpha+\mu)^{q}\right)-\log (\delta)(\mu+\alpha)-q(\mu+\alpha)(\log (\mu+\alpha)-1)+\text { const. } \\
& =-q \alpha \log (\mu+\alpha)+q(\mu+\alpha)+\text { const. }
\end{aligned}
$$

Inserting this in the full Kähler potential (3.7) and using (3.31), we get (up to an additive constant that we forget from now on)

$$
\mathscr{K}=\sum_{k=1}^{s-1}\left(a_{k}-\alpha\right) c_{k} \log t_{k}+B\left(\sum_{m=0}^{q-1}\left|x_{m}\right|^{2}\right) \prod_{k} t_{k}^{\frac{c_{k}}{q}}+\cdots, \quad B=\frac{q}{\delta^{\frac{1}{q}}} .
$$

Here we have introduced the coordinates $\left(x_{0}, \ldots, x_{q-1}\right)$ on the $\mathbb{C}^{q}$ fiber. They are related to $(u, \vec{z})$ via $x_{0}=u^{\frac{1}{q}}, x_{i}=u^{\frac{1}{q}} z_{i} \quad(i>0)$. This procedure changes the periodicity of $\arg (u)$ w.r.t the metric on $X_{K}$ in complete analogy to the Pando ZayasTseytlin/Candelas-de la Ossa metrics discussed in Sect. 2 (keeping the original periodicity would result in a $\mathbb{C}^{q} / \mathbb{Z}_{q}$-singularity).

As we will now see, the above formula provides a Kähler potential in the vicinity of the zero section $\mathscr{F}_{n_{1}, \ldots, n_{m}} \subset X_{V}$. The zero section is given by the equations $x_{m}=0$, $m=0, \ldots q-1$. 
Introducing the holomorphic connection $\widehat{A}=\sum_{k} \frac{c_{k}}{q} \partial \log \left(t_{k}\right)$, we may write out explicitly the line element corresponding to the above potential, in the limit $x_{m} \rightarrow 0$ :

$$
d s^{2}=\sum_{k=1}^{s-1} c_{k}\left(a_{k}-\alpha\right) d s_{k}^{2}+\prod_{k} t_{k}^{\frac{c_{k}}{q}} \sum_{m=0}^{q-1}\left|d x_{m}+\widehat{A} x_{m}\right|^{2}+\cdots
$$

The second term gives a metric in the fiber of the rank- $q$ vector bundle $V=$ $\frac{K_{\mathscr{F}}^{1 / q} \oplus \cdots \oplus K_{\mathscr{F}}^{1 / q} \text {. }}{q \text { times }}$

At the expense of introducing a non-holomorphic complex coordinate $\tau_{m}=$ $\left(\prod_{k} t_{k}^{\frac{c_{k}}{q}}\right)^{1 / 2} x_{m}=\rho_{m} e^{i \phi_{m}}$, we may rewrite (3.34) more compactly:

$$
\begin{aligned}
d s^{2} & =\sum_{k=1}^{s-1} c_{k}\left(a_{k}-\alpha\right) d s_{k}^{2}+\sum_{m=0}^{q-1}\left|d \tau_{m}+i \operatorname{Im}(\widehat{A}) \tau_{m}\right|^{2}+\cdots \\
& =\sum_{k=1}^{s-1} c_{k}\left(a_{k}-\alpha\right) d s_{k}^{2}+\sum_{m=0}^{q-1}\left(d \rho_{m}^{2}+\rho_{m}^{2}\left(d \phi_{m}+\operatorname{Im}(\widehat{A})\right)^{2}\right)+\cdots
\end{aligned}
$$

The absence of a conical singularity at $\rho_{m}=0$ implies that $\phi_{m}$ is periodic in the segment $[0,2 \pi]$.

Acknowledgements Open Access funding provided by Projekt DEAL. We would like to thank D. Lüst and A. A. Slavnov for support and D. Ageev, O. Biquard, P. Gauduchon, S. Gorchinskiy, D. Kaledin, M. Nitta, K. Shramov, P. Zinn-Justin for useful discussions. The research of I.A. was supported by the IMPRS program of the MPP Munich.

Open Access This article is licensed under a Creative Commons Attribution 4.0 International License, which permits use, sharing, adaptation, distribution and reproduction in any medium or format, as long as you give appropriate credit to the original author(s) and the source, provide a link to the Creative Commons licence, and indicate if changes were made. The images or other third party material in this article are included in the article's Creative Commons licence, unless indicated otherwise in a credit line to the material. If material is not included in the article's Creative Commons licence and your intended use is not permitted by statutory regulation or exceeds the permitted use, you will need to obtain permission directly from the copyright holder. To view a copy of this licence, visit http://creativecommons.org/licenses/by/4.0/.

Publisher's Note Springer Nature remains neutral with regard to jurisdictional claims in published maps and institutional affiliations.

\section{A. Complex Structures on Flag Manifolds}

We start from the unitary parametrization (1.3) of the flag manifold, which we schematically write as $\mathscr{F}_{n_{1}, \ldots, n_{s}}=\frac{G}{H}$. The corresponding Lie algebra decomposition is $\mathfrak{g}=\mathfrak{h} \oplus \mathfrak{m}$. We denote $\mathfrak{t}:=i \mathcal{Z}(\mathfrak{h})$, where $\mathcal{Z}(\mathfrak{h})$ is the centralizer of $\mathfrak{h}$ in $\mathfrak{g}$. It is furnished by matrices of the form

$$
\mathfrak{t}=\left\{\operatorname{Diag}(\underbrace{q_{1}, \ldots, q_{1}}_{n_{1}}, \ldots, \underbrace{q_{s}, \ldots, q_{s}}_{n_{s}})\right\}
$$


A root $\alpha \in \mathfrak{g}_{\mathbb{C}}$ is called a $\mathfrak{t}$-root, if it does not vanish identically on $\mathfrak{t}$. Equivalently, $\alpha \notin \mathfrak{h}_{\mathbb{C}}$. Let $t_{0} \in \mathfrak{t}$ be such that $\alpha\left(t_{0}\right) \neq 0$ for all $\mathfrak{t}$-roots $\alpha$. A $\mathfrak{t}$-root $\alpha$ is positive if $\alpha\left(t_{0}\right)>0$. This leads to the notion of $\mathfrak{t}$-chamber in $t$ containing $t_{0}$ :

$$
C\left(t_{0}\right)=\left\{t \in \mathfrak{t}: \alpha(t)>0 \quad \text { if } \quad \alpha\left(t_{0}\right)>0\right\} .
$$

One has the following result:

Theorem. [14,15] There is a one-to-one correspondence between $\mathbf{t}$-chambers and invariant complex structures on the flag manifold $\frac{G}{H}$.

We fix $t_{0}=\operatorname{Diag}(\underbrace{q_{1}, \ldots, q_{1}}_{n_{1}}, \ldots, \underbrace{q_{s}, \ldots, q_{s}}_{n_{s}})$ where all $q_{i}$ 's are distinct. It is easy to see that the value of any $\mathrm{t}$-root on $t_{0}$ is $q_{i}-q_{j}$ for some $i \neq j$. Therefore the $\mathrm{t}$-chambers are given by the permutations of $\left(q_{1}, q_{2}, \ldots, q_{s}\right)$, and there are $s$ ! of them. Obviously, this corresponds to permutations of the set $\left\{n_{1}, \ldots, n_{s}\right\}$. Moreover, the flag manifold may be written in the form $G L(n, \mathbb{C}) / P_{m_{1}, \ldots, m_{s}}$, where the Lie algebra of the parabolic subgroup $P_{m_{1}, \ldots, m_{s}}$ is determined as follows:

$$
\mathfrak{p}_{m_{1}, \ldots, m_{s}}=\mathfrak{h}_{\mathbb{C}} \oplus_{\alpha_{i}>0} \mathbb{C} \alpha_{i} .
$$

It is easy to see that it stabilizes the flag (1.1) in $\mathbb{C}^{n}$, where the dimensions $m_{k}$ of the linear subspaces are given by partial sums of $\left(n_{1}, \ldots, n_{s}\right)$, calculated using the specified ordering. Another interpretation of this result, which uses purely the unitary parametrization (1.3), may be found in [44].

\section{B. Calculation of the Determinant of the Metric}

The goal of this section is to compute the determinant of the Hermitian metric corresponding to the line element (3.10).

First we notice that it is easy to factor out the pieces corresponding to the $u$-dependence of the metric, as well as to the $\mathbb{C P}^{q-1}$ directions. As a result we get

$$
\operatorname{det} g=\frac{1}{|u|^{2} H_{\mu \mu}} \frac{(q(\alpha+\mu))^{q-1}}{\left(1+|\vec{z}|^{2}\right)^{q}} \operatorname{det}\left(g_{\text {red }}^{(\mu)}\right),
$$

where $g_{\text {red }}^{(\mu)}$ is the reduced Hermitian metric, with the line element

$$
d s_{\mathscr{F}}^{2}=\sum_{k=1}^{s-1} c_{k}\left(a_{k}+\mu\right) \pi_{k}^{*}\left(d s_{k}^{2}\right) .
$$

This is a family of metrics on the flag manifold, depending on $\mu$ as a parameter. We will calculate the determinant by studying the set of values of $\mu$, for which $g_{\text {red }}^{(\mu)}$ degenerates. Since the flag manifold is a homogeneous space, and the metric is a $S U(n)$-invariant tensor, $\operatorname{rank}\left(g_{\text {red }}^{(\mu)}\right)$ is the same at every point. To calculate it, we consider the open set in $\mathscr{F}$, where the matrix $W$ introduced in (1.8), which represents the quotient $G l(n, \mathbb{C}) / P$, may be brought to lower-block-triangular form. Evaluating $\pi_{k}^{*}\left(d s_{k}^{2}\right)$ at the point $W=1$, we obtain:

$$
\left.\pi_{k}^{*}\left(d s_{k}^{2}\right)\right|_{W=1}=\sum_{j=m_{k}+1}^{n} \sum_{i=1}^{m_{k}}\left|d w_{j i}\right|^{2} .
$$


Thus

$$
\begin{aligned}
\left.d s_{\mathscr{F}}^{2}\right|_{W=1} & =\sum_{k=1}^{s-1} c_{k}\left(a_{k}+\mu\right) \sum_{j=m_{k}+1}^{n} \sum_{i=1}^{m_{k}}\left|d w_{j i}\right|^{2} \\
& =\sum_{i=1}^{s-1} \sum_{j=i+1}^{s}\left(\sum_{k=i}^{j-1} c_{k}\left(a_{k}+\mu\right)\right) \sum_{a=m_{j-1}+1}^{m_{j}} \sum_{b=m_{i-1}+1}^{m_{i}}\left|d w_{a b}\right|^{2} .
\end{aligned}
$$

The metric at $W=1$ is therefore diagonal, with eigenvalues being equal to $\sum_{k=i}^{j-1} c_{k}\left(a_{k}+\right.$ $\mu$ ), each of multiplicty $n_{i} n_{j}$. At an arbitrary point $W \in \mathscr{F}$, the Hermitian metric $g_{\text {red }}^{(\mu)}$ is represented by a matrix of size $\operatorname{dim}_{\mathbb{C}} \mathscr{F} \times \operatorname{dim}_{\mathbb{C}} \mathscr{F}$, whose entries are linear in $\mu$. Since $\sum_{i<j} n_{i} n_{j}=\operatorname{dim}_{\mathbb{C}} \mathscr{F}$, we get

$$
\operatorname{det}\left(g_{\text {red }}^{(\mu)}\right)=\xi\left(\left\{w_{m i}, \bar{w}_{m i}\right\}\right) f(\mu),
$$

where

$$
f(\mu)=\left.\operatorname{det} g_{\text {red }}^{(\mu)}\right|_{W=1}=\prod_{1 \leq i<j \leq s}\left(\sum_{k=i}^{j-1}\left(a_{k}+c_{k} \mu\right)\right)^{n_{i} n_{j}}
$$

and $\xi\left(\left\{w_{m i}, \bar{w}_{m i}\right\}\right)$ is independent of $\mu$. To find $\xi$, we notice that in the limit $\mu \rightarrow \infty$ the metric (B.2) behaves as $g_{\text {red }}^{(\mu)} \rightarrow \mu g_{K E}$, where $g_{K E}$ is the Kähler-Einstein metric on $\mathscr{F}$ described in (3.6). Since its Kähler potential is $\sum c_{k} \log t_{k}$, from Ric $=g_{K E}$ we find

$$
\xi\left(\left\{w_{m i}, \bar{w}_{m i}\right\}\right)=\left|\kappa\left(\left\{w_{m i}\right\}\right)\right|^{2} e^{-\sum c_{k} \log t_{k}}=\left|\kappa\left(\left\{w_{m i}\right\}\right)\right|^{2} \frac{1}{\prod_{k} t_{k}^{c_{k}}} .
$$

\section{References}

1. Besse, A.: Einstein Manifolds. Springer, Berlin (2008)

2. Eguchi, T., Hanson, A.J.: Asymptotically flat selfdual solutions to Euclidean gravity. Phys. Lett. B 74, 249 (1978)

3. Gibbons, G., Hawking, S.: Gravitational multi-instantons. Phys. Lett. B 78, 430 (1978)

4. Candelas, P., de la Ossa, X.C.: Comments on conifolds. Nucl. Phys. B 342, 246-268 (1990)

5. Pando Zayas, L.A., Tseytlin, A.A.: 3-branes on spaces with $R \times S^{2} \times S^{3}$ topology. Phys. Rev. D 63, 086006 (2001). arXiv:hep-th/0101043 [hep-th]

6. Stenzel, M.B.: Ricci-flat metrics on the complexification of a compact rank one symmetric space. Manuscr. Math. 80(1), 151-163 (1993)

7. Nitta, M.: Noncompact Calabi-Yau Metrics from Nonlinear Realizations. arXiv:hep-th/0309004 [hep-th]

8. Apostolov, V., Calderbank, D.M., Gauduchon, P.: Hamiltonian 2-forms in Kähler geometry. I: general theory. J. Differ. Geom. 73(3), 359-412 (2006)

9. Chen, W., Lu, H., Pope, C.: Kerr-de Sitter black holes with NUT charges. Nucl. Phys. B 762, 38-54 (2007). arXiv:hep-th/0601002 [hep-th]

10. Nakajima, H.: Instantons on ALE spaces, quiver varieties, and Kac-Moody algebras. Duke Math. J. 76(2), 365-416 (1994)

11. Biquard, O., Gauduchon, P.: Hyperkähler metrics on cotangent bundles of hermitian symmetric spaces. Lect. Notes Pure Appl. Math. 184, 287-298 (1996)

12. Calabi, E.: Métriques kählériennes et fibrés holomorphes. Annales scientifiques de l'École Normale Supérieure 4e série, 12(2), 269-294 (1979) 
13. Borel, A., Hirzebruch, F.: Characteristic classes and homogeneous spaces. I. Am. J. Math. 80(2), 458-538 (1958)

14. Alekseevsky, D.: Flag manifolds. Zb. Rad. Mat. Inst. Beograd. (N.S.) 6(14), 3-35 (1997)

15. Alekseevsky, D., Perelomov, A.: Invariant Kähler-Einstein metrics on compact homogeneous spaces. Funktsional. Anal. i Prilozhen. 20, 1-16 (1986). Funct. Anal. Appl. 20(3), 171-182 (1986)

16. Correa, E.M., Grama, L.: Calabi-Yau Metrics on Canonical Bundles of Complex Flag Manifolds. arXiv e-prints (2017). arXiv:1709.07956 [math.DG]

17. Calabi, E.: On Kähler manifolds with vanishing canonical class. In: Algebraic Geometry and Topology. A Symposium in Honor of S. Lefschetz, pp. 78-89. Princeton University Press, Princeton (1957)

18. Yau, S.-T.: Calabi's conjecture and some new results in algebraic geometry. Proc. Natl. Acad. Sci. USA 74, 1798-1799 (1977)

19. Yau, S.-T.: On Ricci curvature of a compact Kähler manifold and complex Monge-Ampere equation I. Commun. Pure Appl. Math. 31, 339-411 (1979)

20. van Coevering, C.: Regularity of Asymptotically Conical Ricci-flat Kähler Metrics. arXiv:0912.3946

21. Goto, R.: Calabi-Yau structures and Einstein-Sasakian structures on crepant resolutions of isolated singularities. J. Math. Soc. Jpn. 64(3), 1005-1052 (2012). https://doi.org/10.2969/jmsj/06431005

22. Sparks, J.: Sasaki-Einstein manifolds. Surv. Differ. Geom. 16, 265-324 (2011). arXiv:1004.2461 [math.DG]

23. Bykov, D.: The geometry of antiferromagnetic spin chains. Commun. Math. Phys. 322, 807-834 (2013). arXiv:1206.2777 [hep-th]

24. Nitta, M.: Auxiliary field methods in supersymmetric nonlinear sigma models. Nucl. Phys. B 711, 133162 (2005). arXiv:hep-th/0312025 [hep-th]

25. Donagi, R., Sharpe, E.: GLSM's for partial flag manifolds. J. Geom. Phys. 58, 1662-1692 (2008). arXiv:0704.1761 [hep-th]

26. Azad, H., Kobayashi, R., Qureshi, M.: Quasi-potentials and Kähler Einstein metrics on flag manifolds. J. Algebra 169(2), 620-629 (1997)

27. Azad, H., Biswas, I.: Quasi-potentials and Kähler Einstein metrics on flag manifolds II. J. Algebra 269(2), 480-491 (2003)

28. Bando, M., Kugo, T., Yamawaki, K.: Nonlinear realization and hidden local symmetries. Phys. Rep. 164(4 and 5), 217-314 (1988)

29. van Coevering, C.: Calabi-Yau metrics on canonical bundles of flag varieties. arXiv:1807.07256 [math.DG]

30. Hwang, A., Singer, M.: A momentum construction for circle-invariant Kähler metrics. Trans. Am. Math. Soc. 354, 2285-2325 (2002)

31. Bielawski, R.: Ricci-flat Kähler metrics on canonical bundles. arXiv Mathematics e-prints (2000). arXiv:math/0006144 [math.DG]

32. Martelli, D., Sparks, J.: Resolutions of non-regular Ricci-flat Kahler cones. J. Geom. Phys. 59, 1175-1195 (2009). arXiv:0707.1674 [math.DG]

33. Pedersen, H., Poon, Y.S.: Hamiltonian constructions of Kähler-Einstein metrics and Kähler metrics of constant scalar curvature. Commun. Math. Phys. 136(2), 309-326 (1991)

34. Dancer, A., Wang, M.Y.: Kähler-Einstein metrics of cohomogeneity one. Math. Ann. 312(3), 503-526 (1998). https://doi.org/10.1007/s002080050233

35. Podesta, F., Spiro, A.: Kaehler manifolds with large isometry group. Osaka J. Math. 36(4), 805-833 (1999)

36. Alekseevsky, D., Zuddas, F.: Cohomogeneity one Kähler and Kähler-Einstein manifolds with one singular orbit I. Ann. Glob. Anal. Geom. 52, 99-128 (2017). arXiv:1611.06521 [math.DG]

37. Alekseevsky, D., Zuddas, F.: Cohomogeneity one Kaehler and Kaehler-Einstein manifolds with one singular orbit, II. arXiv e-prints (2019) arXiv:1906.10633 [math.DG]

38. Bykov, D.: Ricci-flat metrics on the cone over $\mathbb{C P}^{2} \# \overline{\mathbb{C P}^{2}}$, arXiv:1712.07227 [hep-th]

39. Abreu, M.: Kähler geometry of toric manifolds in symplectic coordinates. arXiv:math/0004122 [math.DG]

40. Guillemin, V.: Kaehler structures on toric varieties. J. Differ. Geom. 40(2), 285-309 (1994). https://doi. org $/ 10.4310 / \mathrm{jdg} / 1214455538$

41. Lam, K.Y.: A formula for the tangent bundle of flag manifolds and related manifolds. Trans. Am. Math. Soc. 213, 305-314 (1975)

42. Iskovskikh, V.A., Prokhorov, YuG: Algebraic geometry V: Fano varieties. In: Parshin, A.N., Shafarevich, I.R., Gamkrelidze, R.V. (eds.) Encyclopaedia of Mathematical Sciences, vol. 47. Springer, Berlin (1999)

43. Brion, M.: Lectures on the geometry of flag varieties. In: Pragacz, P, (ed.) Topics in Cohomological Studies of Algebraic Varieties: Impanga Lecture Notes, pp. 33-85. Birkhäuser, Basel (2005). https://doi. org/10.1007/3-7643-7342-3_2 
44. Bykov, D.: Flag manifold $\sigma$-models: the $\frac{1}{N}$-expansion and the anomaly two-form. Nucl. Phys. B 941, 316-360 (2019). arXiv:1901.02861 [hep-th]

Communicated by N. Nekrasov 